\title{
Neonatal ventilator-associated pneumonia:An underdiagnosed problem in the neonatal intensive care units
}

\begin{abstract}
Ventilator-associated pneumonia (VAP) is one of the most common healthcare-associated infections among ventilated neonates. Remarkably, since there are no specific diagnostic criteria for the neonatal period, VAP criteria for infants $<12$ months have been generally applied to the newborn patient. The reported rate of VAP in neonates ranges from 2.7 episodes to 10.9 episodes per 1000 ventilator days and occurs more in preterm babies. Recognized risk factors of VAP in adults and children differ between studies; however Low birthweight, duration of mechanical ventilation, frequent suctioning, and reintubation have been consistently reported. Educational interventions and efforts to improve adherence to strategies of VAP for children have been associated with decreased VAP rates. This minireview aims to give an understanding of the epidemiology, pathogenesis, diagnosis, and treatment of neonatal VAP.
\end{abstract}

Volume 7 Issue 3 - 2017

\section{Saleh Al-Alaiyan, Abdulaziz Binmanee}

Neonatology Section, Department of Pediatrics, King Faisal

Specialist Hospital and Research Centre, Riyadh, Saudi Arabia

\begin{abstract}
Correspondence: Saleh Al-Alaiyan, MD, FRCPC, Neonatology Section, Department of Pediatrics (MBC-58), King Faisal Specialist Hospital and Research Centre, P.O. Box 3354, Riyadh I I I I, Saudi Arabia, Tel 966-I I-442-776 I,; Fax 966-I I-442-7784, Email alaiyan@kfshrc.edu.sa
\end{abstract}

Received: September 24, 2017 | Published: November 01, 2017

\section{Introduction}

Ventilator-associated pneumonia (VAP) is a common and serious problem among mechanically ventilated neonates. It is the second most common hospital-acquired infection among pediatric and NICU patients. ${ }^{1}$ The Centers for Disease Control and Prevention (CDC) defines ventilator-associated pneumonia (VAP) as an episode of pneumonia in a patient who requires a device to assist or control respiration through a tracheostomy or endotracheal tube within 48 hours before the onset of the infection. ${ }^{2}$ It is associated with longer durations of ICU stay and high rates of morbidity and mortality. Unfortunately, few studies have focused on neonates, particularly those with a low or very low birth weight. The incidence of neonatal VAP is difficult to be correctly determined, because it is difficult to differentiate between new or progressive radiographic infiltrates due to neonatal pneumonia or due to exacerbation of bronchopulmonary dysplasia and frequent episodes of atelectasis.

A report from the International Healthcare-associated Infection Control Consortium collected data on healthcare-associated infections from ICUs worldwide from 2007 to 2012, showed that the mean VAP rate in low birth infants at Level III ICUs was 8.95 episodes per 1000 ventilator days. ${ }^{3}$ VAP occurs in higher rates among extreme low birth infants and represents a significant risk factor for complications and death (OR: $3.4 ; 95 \%$ CI: 1.20 to 12.31$).{ }^{4}$ A report from German surveillance system for very-low-birthweight infants. The surveillance included 8677 very low birth weight infants from 52 neonatology departments in Germany. They found that $41.4 \%$ of the neonates who developed healthcare-associated infections weighed $<1 \mathrm{~kg} .{ }^{5}$ In developing countries, the reported rates are significantly higher, ranging from 16.1 to 89 episodes per 1,000 ventilator days. ${ }^{6-10}$

The pathogenesis of VAP in neonates is not well determined, however, Garland ${ }^{9}$ describes the possible sources of microorganisms and the pathogenic mechanisms by which they may cause VAP. The author believes that organisms responsible for VAP can originate from endogenous or exogenous sources. endotracheal intubation leads to impairment of mucociliary clearance and the potential for colonization of the endotracheal tube and trachea, from both endogenous and exogenous sources, which may then descend further and result in pneumonia. Exogenous sources include transmission of infection from a health care workers' hands, contamination of suction apparatus, airway circuits, humidifiers, etc.

In this mini-review, the reader will be able to know the risk factors, methods used to diagnose the problem, the most common organisms responsible for neonatal VAP, the treatment and the recommended steps to prevent neonatal VAP.

\section{Risk factors of neonatal VAP}

Several risk factors have been identified to be related to neonatal VAP. Respiratory diseases are very common among neonates that require mechanical ventilation for long period. Duration of mechanical ventilation has been found to be an important risk factor. In a retrospective cohort study that was conducted on 259 patients who developed 52 episodes of VAP, Yuan et al. ${ }^{11}$ found that re-intubation, duration of mechanical ventilation, treatment with opiates and endotracheal suctioning were significant risk factors for neonatal VAP. In a meta-analysis of observational studies, ${ }^{12}$ a total of eight studies including 370 cases and 1,071 controls were identified. Authors found ten risk factors that were related to neonatal VAP. The following were the risk factors listed in order by odds ratios (ORs): length of stay in NICU (OR 23.45), reintubation (OR 9.18), enteral feeding (OR 5.59), mechanical ventilation (OR 4.04), transfusion (OR 3.32), low birth weight (OR 3.16), premature infants (OR 2.66), parenteral nutrition (OR 2.30), bronchopulmonary dysplasia (OR 2.21), and tracheal intubation (OR 1.12). Moreover, Apisarnthanarak et al. ${ }^{4}$ found a strong association between VAP and mortality among infants who stayed in the NICU $>30$ days (relative risk: $8.0 ; 95 \%$ CI: 1.9-35.0). In addition, they found that infants with VAP also had prolonged NICU length of stay (median: 138 vs 82 days).

Cernada et al. ${ }^{13}$ listed several risk factors of neonatal VAP including; Low birthweight, prolonged mechanical ventilation, opiate treatment for sedation, frequent suctioning and reintubation, bloodstream infection, and steroid use have all been noted to be associated with increased risk of VAP. Low birth infants have immature immune system that places them at an increased risk of nosocomial infection. 
Additionally, their skin and mucous membranes are more permeable and are less effective barriers.

\section{Diagnostic Criteria}

Accurate diagnosis of neonatal VAP is essential to initiate the appropriate treatment. The CDC's NHSN VAP criteria included radiographic, clinical, and microbiologic elements. ${ }^{2}$

The following are the CDC's NHSN VAP criteria for VAP in infants younger than 1 year:

\section{Radiological signs}

Patient with one or more (in patients with underlying diseases two or more) chest $\mathrm{X}$-rays with one of the following findings:

\author{
- new or progressive and persistent infiltrate \\ - consolidation \\ - cavitation \\ - pneumatoceles
}

\section{Clinical signs and symptoms}

Worsening of gas exchange[e.g. oxygen desaturations (e.g. pulse oximetry $<94 \%$ ), increased oxygen requirements, or increased ventilation demand]and three of the following:

- temperature instability with no other recognized cause

- leukopenia $(<4,000 \mathrm{WBC} / \mathrm{mm} 3)$ or leukocytosis $(>15,000 \mathrm{WBC} /$ $\mathrm{mm} \mathrm{3}) *$ and left shift (>10\% band forms)

- new onset of purulent sputum, or change in the character of sputum, or increase in respiratory secretions, or increased suctioning requirements

- apnea, tachypnea, nasal flaring with retraction of chest wall or grunting

- wheezing, rales, or rhonchi

- cough

- bradycardia ( $<100$ beats/min) or tachycardia ( $>170$ beats/min)

Microbiological findings

At least one of the following:

- positive pleural fluid culture

- positive quantitative culture from a minimally contaminated LRT specimen[e.g. BAL $(\geq 104 \mathrm{CFU} / \mathrm{ml})$ * or protected specimen brushing $(\geq 103 \mathrm{CFU} / \mathrm{ml})]$

- $\geq 5 \%$ BAL-obtained cells contain intracellular bacteria on direct microscopic examination (e.g. Gram stain)

- histopathological exam shows at least one of the following criteria for pneumonia: abscess formation or foci of consolidation with intense PMN accumulation in bronchioles and alveoli, positive quantitative culture of lung parenchyma ( $\geq 104 \mathrm{CFU} / \mathrm{g}$ tissue), or evidence of lung parenchyma invasion by fungal hyphae or pseudohyphae

$* \mathrm{WBC}=$ White blood cells; $\mathrm{CFU}=$ colony-forming units.

Unfortunately, these criteria have not been validated in neonates, and they often overlap with many neonatal respiratory diseases that makes the diagnosis of VAP difficult, particularly in low and very low birth infants. In addition, the difficulty in obtaining noncontaminated samples from the respiratory airways makes the diagnosis uncertain. Specific biomarkers of VAP such as Procalcitonin, Cytokines, the Soluble Form of the Triggering Receptor Expressed on Myeloid Cells, and oxidative stress were used in adult population to differentiate between true pneumonia and colonization, although with inconsistent results. Moreover, tracheal aspirates directly obtained from endotracheal tube is commonly used in the NICU instead of using bronchoalveolar lavage and this technique may represent colonization instead of true infection. Köksal et al. ${ }^{14}$ studied the non-bronchoscopic bronchoalveolar lavage (NB-BAL) to diagnose ventilator-associated pneumonia in 145 intubated neonates. Forty newborns with VAP $(90 \%)$ had positive NB-BAL culture. The sensitivity, specificity, and positive and negative predictive values of NB-BAL fluid culture for VAP diagnosis were $90 \%, 90 \%, 70 \%$, and $97 \%$, respectively. In a prospective study to assess the usefulness of Gram staining of tracheal aspirates in extremely preterm neonates with VAP. Katayama et al. ${ }^{15}$ found that Gram-positive cocci and Gram-negative bacilli were the causative microorganisms in 11 and 38 confirmed episodes of VAP, respectively. Moreover, they performed correlations between Gramstain results and final causative organism findings. They found that Gram staining indicated that gram positive cocci was highly $(100 \%)$ specific for Gram-positive VAP and the sensitivity was $82 \%$. For Gram-negative VAP, sputum Gram stain showed high sensitivity $(100 \%)$ and specificity $(82 \%)$ to Gram-negative bacilli. In their study, the most commonly isolated causative organisms in this study were P. aeruginosa $(n=18 ; 37 \%), K$. pneumoniae $(n=7 ; 14 \%)$, and S. marcescens $(n=7 ; 14 \%)$. Methicillin-resistant and methicillinsusceptible Staphylococcus aureus were each isolated from $5(10 \%)$ patients. Polymicrobial organisms also have been predominantly reported in neonatal VAP. (12) Moreover, Carvalho et al. ${ }^{16}$ concluded that the etiological diagnosis of VAP was considered as definitive when any microorganism was isolated from tracheal aspirates at concentrations of greater than or equal to $103 \mathrm{cfu} \mathrm{ml}^{-1}$.

The most commonly isolated causative organisms in neonatal VAP are Gram-negative bacteria. It was estimated to be ranging from $60 \%$ to $97 \%$ with Pseudomonas, Klebsiella and acinetobacter organisms predominating the positive cultures. ${ }^{17}$ Finally, accurate estimates of the true prevalence of a causative organism are highly needed to confirm the diagnosis of VAP and to initiate the appropriate treatment.

\section{Treatment}

Empirical antibiotic use is widespread in the NICUs. In a retrospective cohort study of 52,061 infants in 127 NICUs across California during 2013, Schulman et al. ${ }^{18}$ found a 40 -fold variation in antibiotic prescribing practices, despite similar burdens of proven infections, NEC, surgical volume and mortality. In addition, they found that in some NICUs, antibiotics were overused.

Prolonged initial empirical antibiotic treatment has been associated with increased rates of necrotizing enterocolitis and death for extremely low birth weight infants. In a report from the Eunice Kennedy Shriver NICHD Neonatal Research Network, multivariable analysis showed associations between longer initial empirical antibiotic courses and mortality and morbidities among 4039 extremely low birthweight neonates who survived $>5$ days, received initial empirical antibiotic treatment, and had sterile cultures through the first 3 postnatal days. ${ }^{19}$

Initial antibiotic therapy is empirical and targeted against the most likely organisms, based on the available epidemiological information on the pathogen profile in the neonatal unit and community. As soon as culture and antibiotic susceptibility results are available., antibiotics should be narrowed down or modified. 


\section{Prevention of neonatal VAP}

VAP care bundle, initially developed for the adult population ${ }^{20}$ with positive results in preventing VAP and subsequently it has been modified for neonatal and pediatric populations. ${ }^{8}$ Unfortunately, few of the recommended interventions for adult VAP bundle are not applicable for neonates such as deep vein prophylaxis and gastric ulcer prophylaxis. Sedation vacations is usually recommended in adult VAP bundles to be more accurate in assessing extubation readiness. In the NICUs, sedation vacation is uncommon.

Additionally, some of the recommended interventions are not formally studied in neonates. Semirecumbent body position in intensive-care patients has been shown to decrease frequency and risk of nosocomial pneumonia by reducing gastro-esophageal reflux and subsequent aspiration. ${ }^{21}$ In neonates, there is no strong evidence to support positioning the head of the bed up or a horizontal left or right lateral position, this needs to be investigated. The newborns should remain with head between $20^{\circ}$ to $30^{\circ}$ degrees, unlike pediatric patients with angles between $30^{\circ}$ and $45^{\circ}$, because neonatal beds allow for elevation to a maximum of $30^{\circ} .22$

Closed suction systems are increasingly replacing open suction systems to perform endotracheal toilet in mechanically ventilated intensive care unit patients. In a meta-analysis that involved 1,272 patients, showed no evidence to prefer either of them..$^{23}$ Cordero et al. ${ }^{24}$ compared a closed (group 1) with an open endotracheal suction system (group 2) in premature infants. There were 67 and 66 infants in group 1 and group 2 respectively. They found that $39 \%$ of infants in the closed group and $44 \%$ of infants in the open group became airway colonized with Gram-negative bacilli; differences were statistically significant. There was no difference in terms of VAP or bloodstream infection rates between the two groups. Nevertheless, there is some evidence that closed endotracheal suction is associated with reduction in episodes of hypoxia and bradycardia. ${ }^{25}$ In adults, silver-coated endotracheal tube has been shown to reduce VAP especially during the first 10 days of mechanical ventilation. ${ }^{2}$. Silver-coated endotracheal tubes are not available for neonates. In a randomized controlled clinical study on adult patients, authors used endoclear device for ETT cleaning. Authors found that treated tubes showed reduced mucus accumulation $(0.56 \pm 0.12$ vs $0.71 \pm 0.28 \mathrm{~mL} ; \mathrm{P}=.004)$ and reduced occlusion $(6.3 \pm 1.7$ vs $8.9 \pm 7.6 \% ; \mathrm{P}=.039) .{ }^{27}$ This device has not been tried in neonates.

Other interventions to prevent VAP in adults but no information to include them in the neonatal VAP bundle include; histamine 2 receptor antagonists or antacids, selective decontamination and probiotics. ${ }^{13}$ Strategies to prevent neonatal VAP include staff education, transmission precautions, and bundle practices. Staff education and routine hand washing are very important strategies to ensure compliance and reduce nosocomial infections. Won et al. ${ }^{28}$ conducted a multimodal campaign for hand hygiene promotion in the NICU. The campaign consisted of formal lectures, written instructions and posted reminders regarding hand hygiene and proper handwashing techniques, covert observation, financial incentives, and regular group feedback on compliance. They found that the overall compliance with hand hygiene improved from $43 \%$ at baseline to $80 \%$ during the promotion program. Furthermore, the rate of nosocomial infections decreased from 15.13 to 10.69 per 1,000 patient-days ( $P$ $=.003$ ) and the respiratory tract infections decreased from 3.35 to 1.06 per 1,000 patient-days $(\mathrm{P}=.002)$.

The quality-of-evidence of these interventions diverges from low to good impact on VAP rates. ${ }^{29}$ We can conclude that the following recommended interventions show clear benefit when applied in the NICU setting:

\section{- Hand hygiene}

- Wearing gloves when in contact with secretions

- Minimizing days of ventilation by daily evaluation for readiness to be extubated to nasal continuous airway pressure

- Preventing unplanned extubation by creating a uniform procedure for securing endotracheal tubes and avoid reintubation

\section{- Suctioning orophaynx}

- Preventing gastric distension

- Changing ventilator circuit only when visibly soiled or malfunctioning

- Removing condensate from ventilator circuit frequently

In contrast, the following recommended interventions don't have clear benefit:

- Oral care with antiseptic or colostrum

- Elevation of head of bed 300-450

- In-Line (closed) suctioning

The ventilator bundles are available in many units, but not totally implemented. Prevention of VAP can be achieved in settings with high levels of compliance. In a survey ${ }^{30}$ that included 250 hospitals $(57 \%$ response rate) with 415 ICUs participated. Authors concluded that having a policy in place is insufficient to reduce VAP rates. Monitoring bundle compliance and implementing interventions to ensure high compliance are highly required to decrease rates of VAP. Education programs for implementing VAP are very important to promote adherence to the recommended guidelines. Educational programs should include self-study modules, repeated lectures, workshops] and several brief or standardized sessions. In a systematic review, Jansson et al. ${ }^{31}$ showed that following educational interventions, $66.7 \%$ of the included studies found that the incidence of VAP decreased significantly.

It is important to promote research to include all interventional items affecting the outcome. Additionally, it is recommended to reward the NICU staff who continuously support the adherence to the implemented protocols to reduce the neonatal VAP in their units.

\section{Conclusion}

VAP remains a serious NICU problem among mechanically ventilated neonates that is associated with morbidity and mortality. Neonatal VAP is under-recognized in the NICU because of unspecific signs and symptoms, the overlap with many neonatal respiratory diseases and the difficulty in obtaining non-contaminated samples from the respiratory airways. In addition, the neonatal VAP bundle was not designed for neonates particularly low birth infants, but it was modified from adult and pediatric VAP which were not based on the neonatal pathophysiology. Many of the recommended interventions in neonatal VAP lack a strong evidence. The NICU caregivers should have high index of suspicions of VAP when a ventilated neonate experiences sudden deterioration in his or her ventilatory requirements. Continuing education of NICU caregivers will increase their awareness about the disease and their compliance in implementing the approved guidelines and protocols in their units. There is an urgent need for trials to identify reliable tests that confirm 
the diagnosis of VAP such as biomarkers to initiate treatment without delay.

\section{Acknowledgments}

None.

\section{Conflicts of interest}

The authors declare no conflicts of interest.

\section{References}

1. Gaynes RP, Edwards JR, Jarvis WR. Nosocomial infections among neonates in high-risk nurseries in the United States. Pediatrics. 1996;98:357-361.

2. Centers for Disease Control and Prevention. Criteria for defining nosocomial pneumonia. 2017

3. VD Rosenthal, DG Maki, Y Mehta, et al. International nosocomial infection control consortium (INICC) report, data summary of 43 countries for 2007-2012. Device-associated module. Am J Infect Control. 2014;42(9):942-956

4. Apisarnthanarak A, Holzmann-Pazgal G, Hamvas A, et al. Ventilatorassociated pneumonia in extremely preterm neonates in a neonatal intensive care unit: characteristics, risk factors, and outcomes. Pediatrics. 2003;112(6 Pt 1):1283-1289.

5. Geffers C, Baerwolff S, Schwab F, et al. Incidence of healthcareassociated infections in high-risk neonates:results from the German surveillance system for very-low-birthweight infants. $J$ Hosp Infect. 2008;68(3):214-221.

6. Azab SF, Sherbiny HS, Saleh SH, et al. reducing ventilator associated pneumonia in neonatal intensive care unit using "VAP prevention Bundle" :a cohort study. BMC Infect Dis. 2015;15:314.

7. Deng C, Li X, Zou Y, et al. Risk factors and pathogen profile of ventilator-associated pneumonia in a neonatal intensive care unit in China. Pediatr Int. 2011;53(3):332-337.

8. Tullu MS, Deshmukh CT, Baveja SM. Bacterial nosocomial pneumonia in paediatric intensive care unit. J Postgrad Med. 2000;46(1):18-22.

9. Garland JS. Strategies to prevent ventilator associated pneumonia in neonates. Clin Perinatol. 2010;37(3):629-43.

10. Kawanishi F, Yoshinaga M, Morita M, et al. Risk factors for ventilatorassociated pneumonia in neonatal intensive care unit patients. Journal of Infection and Chemotherapy. 2014;20(10):627-630.

11. Yuan TM, Chen LH, Yu HM. Risk factors and outcomes for ventilatorassociated pneumonia in neonatal intensive care unit patients. $J$ Perinat Med. 2007;35(4):334-338.

12. Bin Tan, Fan Zhang, Xian Zhang, et al. Risk factors for ventilatorassociated pneumonia in the neonatal intensive care unit:a meta-analysis of observational studies. Eur J Pediatr. 2014;173(4):427-434.

13. Cernada M, Brugada M, Golombek S, et al. Ventilator-Associated Pneumonia in Neonatal Patients:An Update. Neonatology. 2014;105(2):98-107.

14. Köksal N, Hacimustafaoğlul M, Celebi S, et al. Nonbronchoscopic bronchoalveolar lavage for diagnosing ventilator-associated pneumonia in newborns. Turk J Pediatr. 2006;48(3):213-220.
15. Katayama Y, Minami H, Enomoto M, et al. Usefulness of Gram staining of tracheal aspirates in initial therapy for ventilator-associated pneumonia in extremely preterm neonates. Journal of Perinatology. 2010;30(4):270-274.

16. Carvalho EM, Massarollo PC, Levin AS, et al. Comparative study of etiological diagnosis of nosocomial pneumonia. Braz J Infect Dis. 2008;12:67-74.

17. Erfani Y, Rasti A, Janani L. Prevalence of Gramnegative bacteria in ventilator associated pneumonia in neonatal intensive care units:a systematic review and metaanalysis protocol. BMJ Open. 2016;6(10):e012298.

18. Schulman J, Dimand RJ, Lee HC. Neonatal intensive care unit antibiotic use. Pediatrics. 2015;135(5):826-833.

19. Cotten CM, Taylor S, Stoll B, et al. Prolonged duration of initial empirical antibiotic treatment is associated with increased rates of necrotizing enterocolitis and death for extremely low birth weight infants. Pediatrics. 2009;123(1):58-66.

20. Institute for Healthcare Improvement . What is a bundle. 2011.

21. Drakulovic MB, Torres A, Bauer TT, et al. Supine body position as a risk factor for nosocomial pneumonia in mechanically ventilated patients:a randomised trial. Lancet. 1999;354(9193):1851-1858.

22. Brierley J, Highe L, Hines S, et al. Reducing VAP by instituting a care bundle using improvement methodology in a UK paediatric intensive care unit. Eur J Pediatr. 2012;171(2):323-330.

23. Jongerden IP, Rovers MM, Grypdonck MH, et al. Open and closed endotracheal suction systems in mechanically ventilated intensive care patients:a meta-analysis. Crit Care Med. 2007;35(1):260-270.

24. Cordero L, Sananes M, Ayers LW. Comparison of a closed (Trach Care $\mathrm{MAC})$ with an open endotracheal suction system in small premature infants. J Perinatol. 2000;20(3):151-156.

25. Taylor JE, Hawley G, Flenady V, et al. Tracheal suctioning without disconnection in intubated ventilated neonates. Cochrane Database Syst Rev. 2011;7(12):CD003065.

26. Tokmaji G, Vermeulen H, Müller MC, et al. Silver-coated endotracheal tubes for prevention of ventilator-associated pneumonia in critically ill patients. Cochrane Database Syst Rev. 2015;12(8):CD009201.

27. Pinciroli R, Mietto C, Piriyapatsom A, et al. Endotracheal Tubes Cleaned With a Novel Mechanism for Secretion Removal:A Randomized Controlled Clinical Study. Respir Care. 2016;61(11):1431-1439.

28. Won SP, Chou HC, Hsieh WS, et al. Handwashing program for the prevention of nosocomial infections in a neonatal intensive care unit. Infect Control Hosp Epidemiol. 2004;25(9):742-746.

29. Michael Klompas M, Richard Branson R, Eric C, et al. Strategies to Prevent Ventilator-Associated Pneumonia in Acute Care Hospitals:2014 Update. Infection Control and Hospital Epidemiology. 2014;35(8):915936

30. Pogorzelska M, Stone PW, Furuya EY, et al. Impact of the ventilator bundle on ventilator-associated pneumonia in intensive care unit. International Journal for Quality in Health Care. 2011;23(5):538-544.

31. Jansson M, Kyngäs H, Kääriainen M. The effectiveness of educational programmes in ventilator bundle implementation:A systematic review. OA Anaesthetics. 2013;1(1):6. 